\title{
Absceso cerebral por Nocardia spp. en pacientes inmunocompetentes. Presentación de 2 casos
}

\author{
(Cerebral Abscess by Nocardia spp. in immunocompetent patients: \\ Report of 2 cases)
}

Novoa R Felipe $^{1 *}$, Vallejo H Gustavo $^{1}$, Kral B Alejandro
Cortés H Jorge
${ }^{1}$, Wilson L Gonzalo

${ }^{1}$ Residente Medicina Interna, Universidad de Valparaíso. ${ }^{2}$ Infectología, Hospital Carlos Van Buren, Valparaíso.

*Autor para correspondencia: felo.novoa@gmail.com

RECIBIDO: 01 de Marzo de 2018

APROBADO: 04 de Abril de 2018

DOI: 10.22370/bolmicol.2018.33.1.1095

LOS AUTORES DECLARAN NO TENER CONFLICTO DE INTERESES

Palabras claves: absceso cerebral; inmunocompetente; Nocardia

Key words: cerebral abscess; inmunocompetent; Nocardia

\section{RESUMEN}

Nocardia corresponde a un género de bacterias gram positivo que puede producir compromiso pulmonar, sistémico y abscesos cerebrales, especialmente en pacientes inmunocomprometidos. La infección cerebral por Nocardia spp es extremadamente infrecuente en pacientes inmunocompetentes, por lo cual se reportan dos casos: caso 1: mujer de 61 años, sana, consulta por cefalea y paresia en hemicuerpo izquierdo. Estudio con TAC y RM de encéfalo demuestran absceso cerebral. Se inició tratamiento con ceftriaxona mas cloxacilina y fue drenado quirúrgicamente. En el cultivo del LCR se aisló Nocardia spp. cambiándose esquema a cotrimoxazol con meropenem por 6 semanas. Caso 2: varón de 72 años, hipertenso y tabáquico crónico. Consultó por cefalea, paresia de extremidad inferior derecha y pérdida de visión de ojo derecho. Estudio con TAC y RM de encéfalo objetiva absceso cerebral parietal izquierdo. Se inició tratamiento con ceftriaxona, metronidazol y vancomicina. Se realizó drenaje quirúrgico. El cultivo de absceso resultó positivo para Nocardia spp, ajustándose esquema a cotrimoxazol y meropenem por 6 semanas. Requirió tratamiento prolongado por presentar lenta regresión clínica e imagenológica.

\section{ABSTRACT}

Nocardia is a gram positive bacterial genus. Is involved in pulmonary, systemic and brain abscess usually in immunocompromised patients. Nocardia spp. brain infection is extremely rare in immunocompetent patients, hereby we report 2 cases: case 1: 61 years old woman, without morbid conditions, consulted for headache and left he- 
miparesis. Study with CT and MRI of encephalon shows brain abscess. Treatment with ceftriaxone plus cloxacilin and surgical drainage were started. In CSF culture, Nocardia spp. was obtained. Scheme was changed to cotrimoxazole with meropenem to complete 6 weeks. Case 2: male of 72 years old, history of smoking and hypertension. Consulted for headache, paresis of right leg and loss of vision of the right eye. CT and MRI showed left parietal brain abscess. Treatment with ceftriaxone, metronidazole and vancomycin were started. Surgical drainage was performed. Abscess culture was positive for Nocardia spp., adjusting scheme to cotrimoxazole and meropenem for 6 weeks. It required prolonged treatment due to slow imaging and clinical regression.

\section{INTRODUCCIÓN}

Nocardia correponde a un género de bacterias gram positivo, que incluye más de 80 especies y suele producir afectación pulmonar o sistémica, comprometiendo generalmente a individuos inmunocomprometidos ${ }^{1}$.

Las infecciones cerebrales por Nocardia farcínica tienen una mortalidad global superior a $20 \%$ en pacientes inmunocomprometidos ${ }^{2}$. Existe poca experiencia en pacientes inmunocompetentes, por lo cual se reportan 2 casos de absceso cerebral causados por este agente.

\section{CASOS CLÍNICOS}

Caso 1: mujer de 61 años, sana, que consulta por un cuadro de 2 semanas de cefalea hemicránea derecha, asociada a decaimiento y anorexia, a lo que se agregó hemiparesia facio-braquio-crural izquierda en los últimos 5 días. Se realizó Tomografía axial computada (TAC) de encéfalo que demostraba proceso expansivo cerebral frontal derecho, con edema perilesional y gran efecto de masa con signos de herniación subfalcina. La TAC de tóraxabdomen y pelvis no presentaba alteraciones.

Durante la hospitalización se realizó Resonancia magnética (RM) de encéfalo (Figura 1) que confirmó la presencia de masa cerebral, sugerente de absceso ( $30 \times 35 \mathrm{~mm}$ ), por lo que se inició tratamiento con ceftriaxona y cloxacilina y se realizó cirugía de drenaje e instalación de derivación ventrículo-externa. Se realiza tinción de gram y cultivo de absceso cerebral (Figura 2). Estudio de VIH no reactivo, con inmunoglobulinas normales.

El cultivo de líquido cefalorraquídeo (LCR) resultó positivo para Nocardia spp., por lo que se cambió esquema a cotrimoxazol forte $(800 / 160) 1$ comprimido vía oral (VO) c/8 hrs. y meropenem 1 gr c/8 hrs. Endovenoso (EV) en infusión estándar para completar 6 semanas.

La evolución clínica fue favorable y se normalizaron los parámetros de actividad inflamatoria. No hubo secuelas ni recaídas durante el seguimiento ambulatorio a 6 meses.

Figura 1. RM de cerebro con gadolinio, corte transversal secuencia T1 post contraste. Se observa absceso cerebral frontal derecho de $30 \times 35 \mathrm{~mm}$, con edema peri lesional, gran efecto de masa y herniación subfalcina. Captación de contraste en anillo.

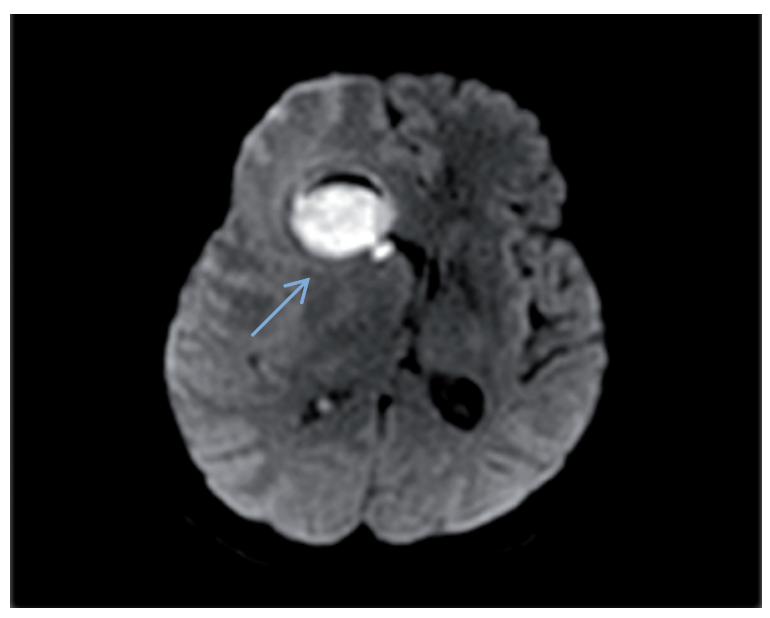


Figura 2. Tinción de Gram de secreción de absceso cerebral. Se observan cocobacilos gram $(+)$ delgados en forma de filamentos ramificados.

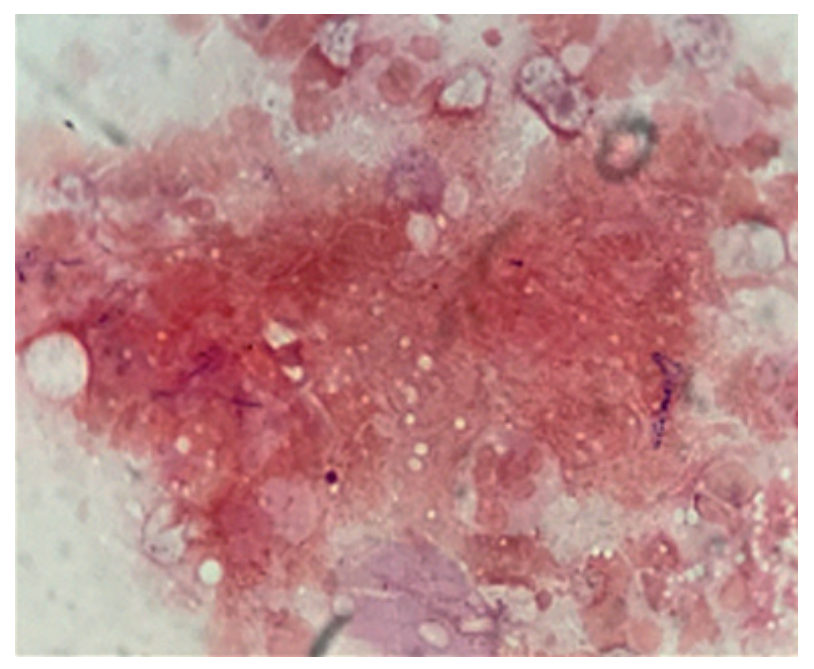

Caso 2: varón de 72 años, hipertenso y tabáquico crónico que consultó por cuadro de decaimiento, agregándose en los últimos días paresia de extremidad inferior derecha, diaforesis, cefalea y pérdida de visión del ojo derecho. Se realizó TAC de encéfalo que evidenció lesión expansiva a nivel parietal izquierdo, con estudio ampliado con RM (Figura 3) con lesión extensa de 30 x $22 \mathrm{~mm}$ con efecto de masa y captación de contraste en anillo, con extensión hacia cortical, infiltrando calota, muy sugerente de absceso cerebral (Figura 4). Se inició terapia con ceftriaxona $2 \mathrm{gr} \mathrm{c} / 12 \mathrm{hrs}$. EV, metronidazol 500mg c/8 hrs. EV, vancomicina 1 gr. c/12 hrs EV y se tomó TAC de tórax, abdomen y pelvis que sólo demostró enfisema pulmonar y nódulos pulmonares apicales, probablemente secuelares a tuberculosis antigua, con baciloscopía de expectoración negativa. Estudio VIH no reactivo e inmunoglobulinas normales.

Se realizó drenaje de absceso cerebral y los cultivos corrientes de secreción y tejido resultaron positivos para Nocardia spp., por lo que se ajustó terapia antimicrobiana a meropenem 1 gr. c/12 hrs. EV (infusión estándar) y cotrimoxazol forte
(800/160) 1 comp./día VO para completar 6 semanas de tratamiento.

Evolucionó con índices de actividad inflamatoria elevados, por lo que se realizó RM de control que mostraba presencia de absceso residual de 27 x $18 \mathrm{~mm}$. a nivel témporo parietal, asociado a colección epidural. Además, informó signos de leptomeningitis supra e infratentorial y de infarto agudo putaminal posterior derecho, por lo que se extiendió la terapia antibiótica por 8 semanas en forma adicional.

El seguimiento imagenológico demostró reducción significativa en el tamaño del absceso, y la presencia de la colección epidural con aspecto de empiema. Fue evaluado en reunión neuroquirúrgica, dada buena evolución clínica se desestima necesidad de nueva intervención.

A los 6 meses de seguimiento no se observaron recaídas, pero mantiene secuela motora en hemicuerpo derecho (paresia M4 armónica).

Figura 3. RM de cerebro con gadolinio, corte transversal, secuencia T1 post contraste. Lesión extensa de 30 x $22 \mathrm{~mm}$ con efecto de masa y captación de contraste en anillo, con extensión hacia cortical, infiltrando calota, sugerente de absceso cerebral.

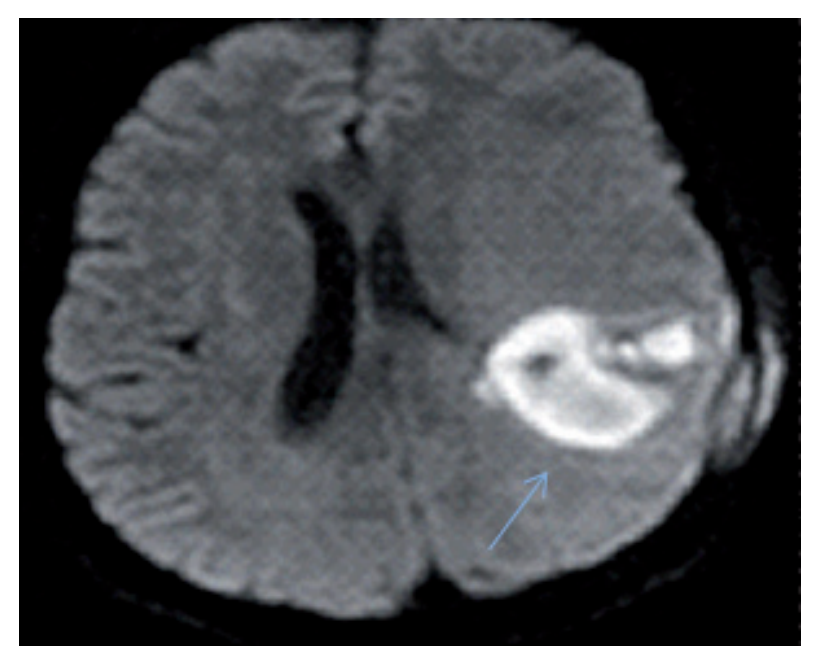


Absceso cerebral por Nocardia spp. en pacientes inmunocompetentes. - Novoa F. et al.

Figura 4. RM de cerebro con gadolinio, corte transversal, secuencia de Perfusión. Se aprecia pared de absceso escasamente vascularizada, restricción de difusión central, contrario a lo observado en tumores cerebrales (diagnóstico diferencial imagenológico).

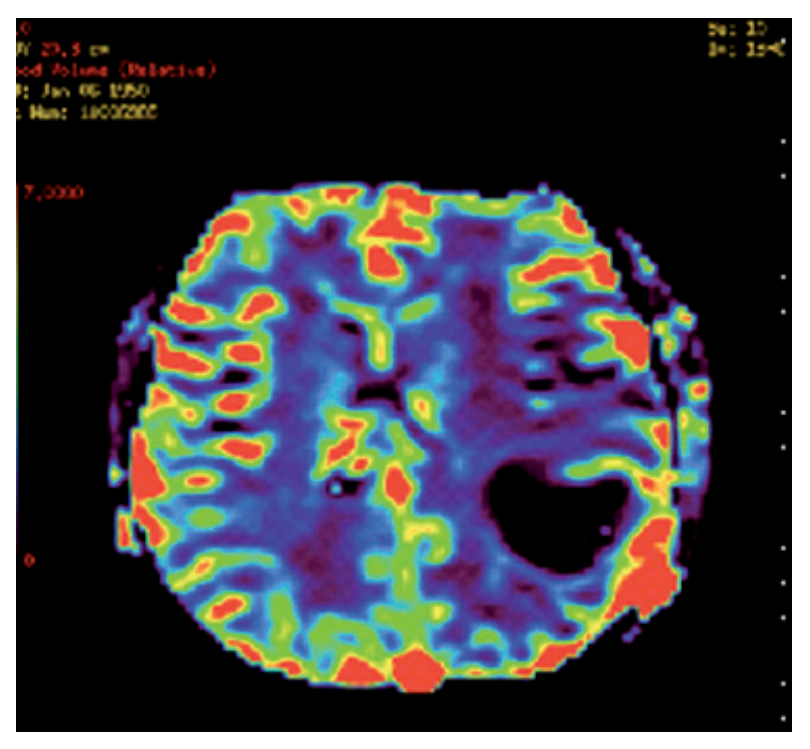

\section{DISCUSIÓN}

En este reporte se describen dos casos de absceso cerebral primario por Nocardia spp. en pacientes inmunocompetetes. La infección de sistema nervioso central por Nocardia spp. es una enfermedad raramente reportada, especialmente en pacientes inmunocompetentes ${ }^{3}$. La especie más frecuentemente asociada a infección cerebral es Nocardia farcinica $^{4}$. El compromiso de sistema nervioso central puede ser meníngeo o en forma de absceso, siendo ocasional confundirlos con neoplasias a la imagenología 5 . Usualmente la vía de infección primaria es por inhalación de aerosoles, que penetran al tracto respiratorio ${ }^{6}$. El drenaje quirúrgico y la toma de cultivos intra-operatorios son el estándar para el tratamiento y diagnóstico definitivo ${ }^{7,8}$. La identificación temprana del microorganismo es perentoria para poder iniciar la antibioticoterapia efectiva. En los escasos casos publicados de absceso cerebral por Nocardia spp. en inmunocompetentes el tratamiento inicial de amplio espectro con combinaciones de carbapenémicos, cotrimoxazol y/o fluoroquinolonas y posteriormente tratamiento prolongado con cotrimoxazol (6-12 meses) ha demostrado resultados favorables ${ }^{9}$. Es fundamental conocer el patrón de susceptibilidad a trimetropimsulfametoxazol. En ambos casos clínicos se pudo obtener cultivo de Nocardia spp. con el adecuado estudio de susceptibilidad a cotrimoxazol, lo que explica los resultados favorables. Con respecto al tratamiento antibiótico inicial se utilizó meropenem en infusión convencional (intermitente). Estudios han demostrado seguridad en el uso de meropenem en infecciones del sistema nervioso central, reportándose convulsiones asociadas a meropenem tan bajo como $0.07 \%$ (muy baja incidencia) ${ }^{10}$. En ambos casos clínicos se utilizaron anticonvulsivantes profilácticos con fenitoína, ajustado con niveles plasmáticos. No se presentaron crisis convulsivas. No existen publicaciones sobre uso de meropenem en infusión extendida en absceso cerebral por Nocardia spp. Extrapolando datos de estudios de meropenem en infusión extendida en infecciones graves por gram (-) y meningitis es presumible que esta estrategia logre adecuada concentración y tiempo sobre la concentración inhibitoria mínima (CIM) en el sistema nervioso central (SNC) ${ }^{11}$. Esta estrategia ha demostrado beneficios, especialmente en tratamientos con beta lactámicos antipseudomonas en $\mathrm{SNC}^{12}$. De acuerdo a la literatura existente, tanto la excisión quirúrgica o aspiración guiada por TC del absceso cerebral ha demostrado efectividad en erradicación de la enfermedad ${ }^{13-14}$. En nuestra experiencia apoyamos la realización de drenaje quirúrgico extenso, debido a que en ambos casos los abscesos fueron de gran tamaño y con efecto de masa, con riesgo de complicaciones por hipertensión endocraneana y herniación subfalcina. Los resultados fueron favorables en lo infeccioso. En el caso 2 la paciente persistió con una secuela neurológica con regresión significativa al control en 6 meses (paresia de hemicuerpo derecho M4 armónica) que no limita las actividades de la vida diaria. No existen estudios que caractericen 
las secuelas neurológicas del absceso cerebral por Nocardia spp., es de esperar que las manifestaciones neurológicas remanentes en este caso disminuyan gradualmente con el tiempo. En una revisión sistémica que evaluó reportes de absceso cerebral, publicados entre 1970 y 2013 demostraron una reducción de la mortalidad por caso de $40 \%$ a $10 \%$ en los últimos 10 años ${ }^{15}$. Las secuelas neurológicas más frecuentes son las convulsiones recurrentes, especialmente en lesiones de lóbulos frontales. Los factores de mal pronóstico al ingreso son la rápida progresión de la infección, compromiso de conciencia profundo, estupor o coma y evacuación intraventricular ${ }^{16}$.

\section{REFERENCIAS}

1. Brown-Elliott BA, Brown JM, Conville PS, Wallace RJ Jr. Clinical and laboratory features of the Nocardia spp. based on current molecular taxonomy. Clin Microbiol Rev. 2006;19(2):259.

\section{Kumar VA, Agustine D, Panikar D, Nandaku-} mar A, Dinesh KR, Karim S, et al. Nocardia farcinica brain abscess: epidemiology, pathophysiology, and literatura review. Surg Infect (Larchmt). 2014;15:640-646.

3. Beaman BL, Beaman L. Nocardia species: host-parasite relationships. Clin Microbiol Rev. 1994;7(2):213.

4. Galacho-Harriero A, Delgado-López PD, Ortega-Lafont MP, Martín-Alonso J, Castilla-Díez JM, Sánchez-Borge B. Nocardia farcinica brain abscess: report of 3 cases. World Neurosurgery. 2017; 106: 1053.e15-.e24.

5. Wilson JW. Nocardiosis: updates and clinical overview. Mayo Clin Proc. 2012; 87: 403-407.

6. Valdezate S, Garrido N, Carrasco G, Medina-Pascual MJ, Villalón P, Navarro AM, et al. Epidemiology and susceptibility to antimicrobial agents of the main Nocardia species in Spain. J Antimicrob Chemother. 2017; 72(3):754.

7. Beaman BL, Beaman L. Nocardia species: host-parasite relationships. Clin Microbiol Rev. 1994; 7:213-264.
8. Aras Y, Sabanci PA, Izgi N, Boyali O, Ozturk O, Aydoseli A, et al. Surgery for Pyogenic Brain Abscess over 30 Years: Evaluation of the Roles of Aspiration and Craniotomy. Turk Neurosurg. 2016; 26:39-47.

9. Bose BB. Diagnosis and treatment of nocardial brain abscess. Neurosurg Q. 2002; 12:182-193.

10. Linden P. Safety Profile of Meropenem: An Updated Review of Over 6000 Patients Treated with Meropenem.[Review]. Drug Safety. 2007; 30(8):657-668.

11. Nicasio AM, Quintiliani R, DeRyke AC, et al. Treatment of Serratia marcescens Meningitis with prolonged infusion of meropenem. Ann Phamacother. 2007;41(6):1077-1081.

12. Bauer KA, West JE, O'Brien JM, et al. Extended-infusion cefepime reduces mortality in patients with Pseudomonas aeruginosa infections. Antimicrob Agents Chemother. 2013; 57(7):2907-2912.

13. Hakan T. Management of bacterial brain abscesses. Neurosurg Focus. 2008;24(6):E4.

14.Torres $O H$, Domingo $P$, Pericas $R$, Boiron $P$, Montiel JA, Vázquez G. Infection caused by $\mathrm{No}^{-}$ cardia farcinica: case report and review. Eur J Clin Microbiol Infect Dis. 2000; 19:205- 212. 
15. Brouwer MC, Coutinho JM, van de Beek D. 16. Seydoux C, Francioli P. Bacterial brain absClinical characteristics and outcome of brain abs- cesses: factors influencing mortality and sequelae. cess: systematic review and meta-analysis. Neuro- Clin Infect Dis. 1992;15(3):394.

logy. 2014;82(9):806. 